\title{
ChemComm
}

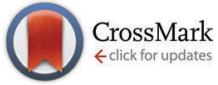

Cite this: Chem. Commun., 2015, 51, 9366

Received 31st March 2015 Accepted 20th April 2015

DOI: $10.1039 / c 5 c c 02675 j$

www.rsc.org/chemcomm

\section{Multiphoton luminescence imaging of chemically functionalized multi-walled carbon nanotubes in cells and solid tumors $\dagger$}

\author{
N. Rubio, ${ }^{a}$ L. M. Hirvonen, $\ddagger^{\mathrm{b}}$ E. Z. Chong, $\ddagger^{\mathrm{b}}$ J. T. W. Wang, ${ }^{a}$ M. Bourgognon, ${ }^{a}$ \\ H. Kafa, ${ }^{a}$ H. A. F. M. Hassan, ${ }^{a}$ W. T. Al-Jamal, ${ }^{C}$ D. McCarthy, ${ }^{d}$ C. Hogstrand, ${ }^{e}$ \\ F. Festy* ${ }^{\text {b }}$ and K. T. Al-Jamal*a
}

The intrinsic nonlinear photoluminescence (PL) property of chemically functionalized multi-walled nanotubes MWNTs (f-MWNTs) is reported in this study. $f-M W N T s$ are imaged in fixed lung epithelial cancer cells (A549) and Kupffer cells in vitro, and in subcutaneously implanted solid tumors in vivo, for the first time, using multiphoton $\mathrm{PL}$ and fluorescence lifetime imaging (FLIM). Multiphoton imaging in the near-infrared excitation region ( 750-950 $\mathrm{nm}$ ), employed in this study in a label-free manner, provides sensitivity and resolution optimal to track f-MWNTs within intra-cellular compartments and facilitates tumour imaging and sentinel lymph node tracking in vivo. Wider applications include employing this technique in live imaging of $\mathrm{f}-\mathrm{MWNT}$ in biological milieu to facilitate image-guided drug delivery.

In biological samples, multiphoton fluorescence microscopy offers noticeable advantages over single-photon fluorescence imaging. The main benefits of this relatively recent technology include reduced phototoxicity outside the focal plane and the use of near-infrared (NIR) excitation wavelengths (700 $\mathrm{nm}$ to $1100 \mathrm{~nm}$ ) to facilitate deep tissue imaging. For this reason, nonlinear photoluminescence (PL) of single-walled nanotubes (SWNTs) has been widely reported. ${ }^{1}$ However, while chemically functionalized multi-walled nanotubes (f-MWNTs) have been extensively studied as delivery tools both in vitro ${ }^{2-4}$ and in vivo ${ }^{5}$ due to their improved dispersibility in aqueous media, only few studies have investigated the nonlinear optical properties of MWNTs. ${ }^{6}$ In this study, we are reporting the intrinsic nonlinear

\footnotetext{
a Institute of Pharmaceutical Science, King's College London,

Franklin-Wilkins Building, 150 Stamford Street, London SE1 9NH, UK.

E-mail: khuloud.al-jamal@kcl.ac.uk

${ }^{b}$ Biomaterials and Biomimetics Department, King's College London Dental Institute, London, SE1 9RT, UK. E-mail: frederic.festy@kcl.ac.uk

'School of Pharmacy, University of East Anglia, Norwich Research Park, Norwich NR4 7TJ, UK

${ }^{d}$ UCL School of Pharmacy, 29-39 Brunswick Square, London, WC1N 1AX, UK

${ }^{e}$ Diabetes and Nutritional Sciences Division, King's College London,

Franklin-Wilkins Building, 150 Stamford Street, London SE1 9NH, UK

$\dagger$ Electronic supplementary information (ESI) available. See DOI: 10.1039/c5cc02675j

$\ddagger$ Equal contribution.
}

PL properties of f-MWNTs with the aim of imaging them in biological environments. We found that f-MWNTs could be excited safely with a femtosecond laser in the 750-950 nm wavelength range with power densities below $10^{6} \mathrm{~W} \mathrm{~cm}^{-2}$. The f-MWNTs were found to be highly photostable with a short excited state lifetime $(<100 \mathrm{ps})$. To demonstrate the imaging potential of these versatile nanocarriers, multiphoton PL lifetime imaging was carried out in lung epithelial cancer cells A549 and Kupffer cells in vitro, and in colon cancer solid tumors.

f-MWNTs were synthesized using 1,3-dipolar cycloaddition ${ }^{7}$ or by amidation reaction. ${ }^{8}$ After the functionalization processes, stable aqueous dispersions of f-MWNTs were obtained (solubility: $1 \mathrm{mg} \mathrm{mL}^{-1}$ ) without the need of surfactants. It is worth mentioning that MWNTs offer higher water-dispersibility compared to SWNTs as the latter require harsher conditions for functionalisation which may disrupt their electronic properties. Chemical structures of the final f-MWNT conjugates are shown in Fig. 1A and Fig. S1A (ESI $\dagger$ ). f-MWNT were characterized by thermogravimetric analysis, ninhydrin assay and transmission electron microscopy (Fig. S1B-D, ESI $\dagger$ ). A standard home-built 2-photon microscope (Fig. 1B) was used for the PL measurements and imaging. f-MWNT in dimethylformamide $\left(1 \mathrm{mg} \mathrm{mL} \mathrm{mL}^{-1}\right)$ were dropcasted on a glass coverslip and left to dry under ambient conditions prior to laser excitation. A 140 fs ultrafast tunable titanium/sapphire laser was used to excite the f-MWNTs and their PL spectra were measured between $400 \mathrm{~nm}$ and $650 \mathrm{~nm}$ (Fig. 1C, left). As the excitation power was increased stepwise from $\sim 3.7 \mathrm{~mW}$ to $\sim 6.7 \mathrm{~mW}$, a progressive change in the PL lineshape was observed. By normalizing all spectra to the intensity at $568 \mathrm{~nm}$, a reduction of the blue band and an increase of the red band are observed as the excitation power increases. A log-log plot of the total emission intensity against excitation power yields a straight line with slopes of $2.7 \pm 0.1$ and $4.8 \pm 0.2$ for excitation wavelengths of 750 and $950 \mathrm{~nm}$ respectively (Fig. 1C, right), indicating the number of photons involved in the non-linear absorption process. A major advantage in using CNTs for imaging is the inherent stability of their PL over time. The bleaching rate of the f-MWNT was measured at $750 \mathrm{~nm}$ excitation wavelength for a total 
A

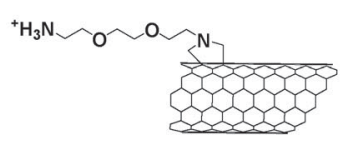

MWNT- $\mathrm{NH}_{3}{ }^{+}$
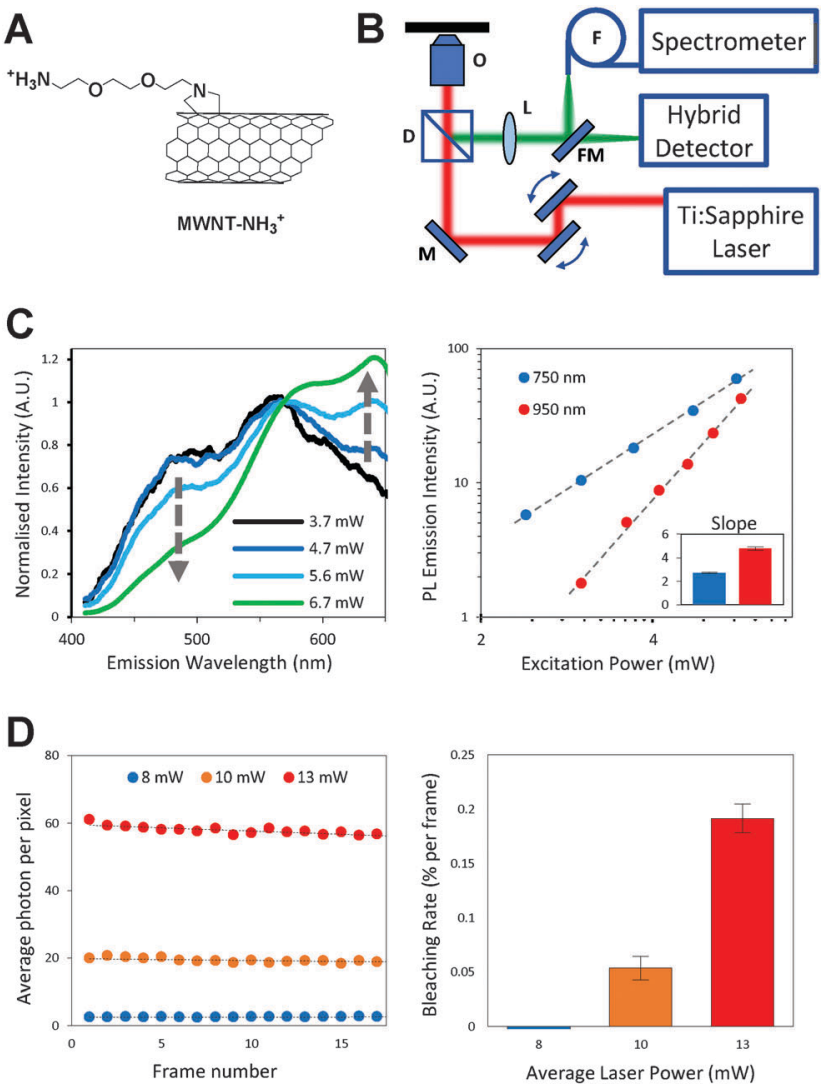

\section{E}
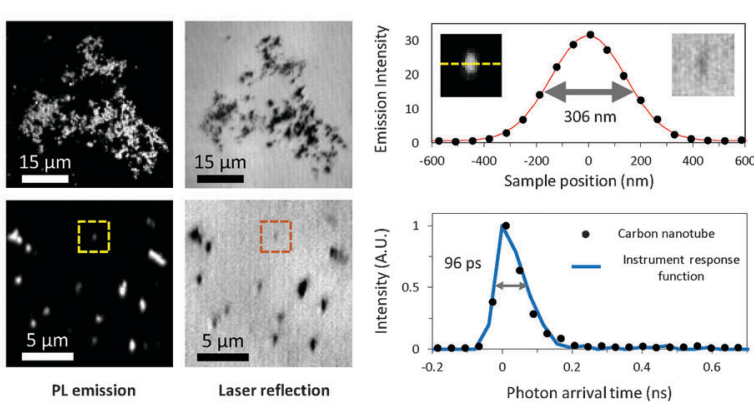

Fig. 1 Optical properties of MWNT- $\mathrm{NH}_{3}{ }^{+}$derivative. (A) Diagram showing the structure of the MWNT- $\mathrm{NH}_{3}{ }^{+}$derivative investigated in this work. (B) Schematic representation of the multiphoton microscope used in this study. (C) Left panel: multiphoton photoluminescence spectra of dropcasted $\mathrm{MWNT}-\mathrm{NH}_{3}{ }^{+}$at $750 \mathrm{~nm}$ excitation using different laser powers. Photoluminescence signals were normalized to $568 \mathrm{~nm}$ emission intensities. Right panel: log-log plot of the photoluminescence intensity as a function of the laser power at both $750 \mathrm{~nm}$ and $950 \mathrm{~nm}$ excitation wavelengths. Slope values were above the value of 2 indicating multiphoton absorption. (D) Left panel: time lapses photobleaching studies of MWNT- $\mathrm{NH}_{3}{ }^{+}$sample carried out at a constant laser power $(8,10$ and $13 \mathrm{~mW}$ ) for a total of 17 lapses (750 nm excitation wavelength). Right panel: corresponding bleaching rates showing the photostability of these nanostructures. (E) Left panel: PL (left) and back-scattered (right) images of MWNT- $\mathrm{NH}_{3}{ }^{+}$of large (top) and small (bottom) aggregates $(750 \mathrm{~nm}$ excitation wavelength). Right panel: $\mathrm{PL}$ line profile over a small aggregate (top) and lifetime transient of the MWNT- $\mathrm{NH}_{3}{ }^{+}$overlaid with the instrument response curve of the microscope.

of 30 frames $(256 \times 256$ pixels, $586 \mathrm{~nm}$ pixel size and $10 \mu$ s dwell time) and was found to be negligible for laser powers up to $13 \mathrm{~mW}$ corresponding to a detector count rate of $\sim 6 \mathrm{MHz}$ (Fig. 1D). Typical images of large (top) and small (bottom) aggregates of drop-casted f-MWNT are shown in Fig. 1E where the smallest PL feature observed has a FWHM of $306 \mathrm{~nm}$, limited by the resolution of the instrument. Similarly, the PL lifetime of the f-MWNT was measured to be $96 \mathrm{ps}$, which is the same as the temporal resolution of the instrument. It is worth noting that both the true size and PL lifetime of these nanocarriers are expected to be much smaller: $0.5-2 \mu \mathrm{m}$ in length, $20-30 \mathrm{~nm}$ diameter and 10-15 ps lifetime.

Carbon nanotubes (CNTs) have been reported to be strong absorbers, efficiently converting IR light to heat, and were used due to this effect for thermal ablation of cancer cells. ${ }^{9,10}$ However, under intense excitation condition, CNTs can be irreversibly damaged. We recorded the Raman D-band and G-band of the f-MWNT ( $1350 \mathrm{~cm}^{-1}$ and $1590 \mathrm{~cm}^{-1}$ respectively) and observed their tubular structure using scanning electron microscopy (SEM) after irradiating them with $10 \mathrm{~mW}$ and $15 \mathrm{~mW}$ laser powers. The focused laser beam was scanned 3 times across $160 \times 160 \mu \mathrm{m}$ area $(256 \times 256$ pixels $)$ at a frame rate of $\sim 1 \mathrm{~Hz}$. While the lower excitation power, mimicking imaging conditions, did not alter the f-MWNT Raman spectrum and structure (Fig. S2A and B, left, ESI $\dagger$ ), imaging at higher power significantly reduced both $\mathrm{D}$ and $\mathrm{G}$ bands while the SEM image showed some noticeable structural damages (Fig. S2A and $\mathrm{B}$, right, ESI $\dagger$ ). It is important to highlight the fact that no absolute laser damage threshold could be established and the f-MWNT absorption characteristic seem to depend on the aggregates' size. However, imaging with excitation power below $10 \mathrm{~mW}$ was reproducibly showing no damages and therefore, was considered to be safe. Moreover, by careful monitoring of the power applied, heat ablation followed by cell kill can be achieved, although this phenomenon was not explored in this study.

f-MWNT were incubated with lung epithelial cells (A549) or Kupffer cells (KC), primary phagocytic cells of the liver, for period ranging from 4 hour to 24 hours, at $37^{\circ} \mathrm{C}$. Cells were fixed and stained with Alexa Fluor ${ }^{\mathbb{R}} 488$ phalloidin (actin filament stain) and DAPI (nuclear stain), then imaged with multiphoton PL microscopy. f-MWNT, actin and nuclei were observed in the red (595-665 nm), green (500-524 nm) and blue (430-490 nm) channels, respectively (Fig. 2A). f-MWNTs could be imaged in both A549 and KC cells and signals were stronger at 24 hours compared to 4 hours incubation. f-MWNTs were localized inside the cells in a time-dependent and temperature-dependent manner, which agrees with our earlier studies using other techniques such as flow cytometry, confocal laser microscopy (using fluorescentlylabeled f-MWNTs) ${ }^{3,11}$ and transmission electron microscopy. ${ }^{3}$ As expected, a higher uptake was seen in KC compared to A549 cells due to the phagocytic nature of the former cell type. A549 cells were incubated with f-MWNTs at $4{ }^{\circ} \mathrm{C}$ for 4 hours (for uptake inhibition) and no signals could be observed (Fig. S3, ESI†). The same results were obtained for f-MWNT functionalized by amidation (Fig. S4, ESI $\dagger$ ).

The CNT aggregate size in cells has been considered by measuring the photoluminescence line profile of the CNT clusters that have been internalized within the cells (Fig. S5, ESI $\dagger$ ). KC showed CNT clusters (Fig. S5A, ESI $\dagger$ ) with a full width 
A
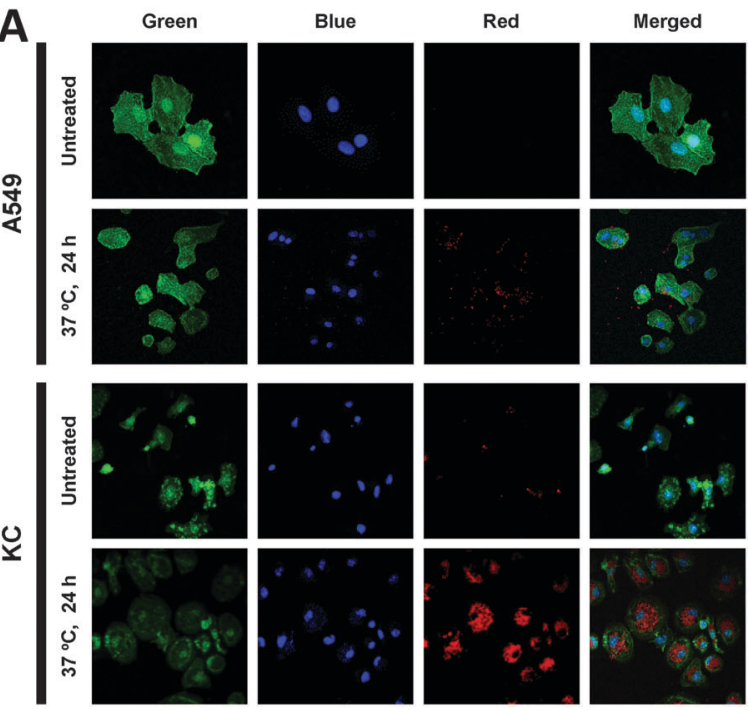

B

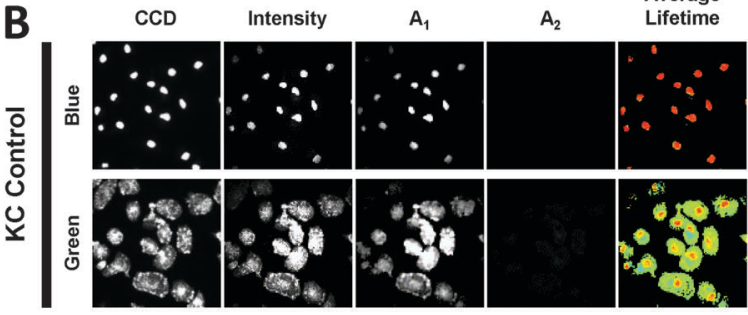

$\mid$

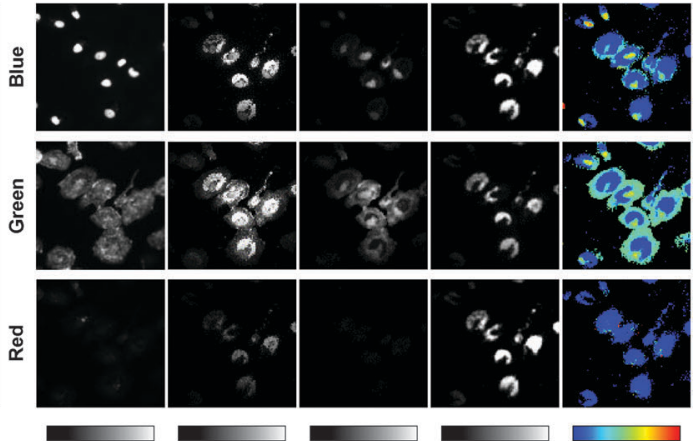

Fig. 2 Multiphoton photoluminescence and lifetime imaging of A549 and Kupffer cells (KC) incubated with MWNT-NH${ }_{3}{ }^{+}$at $20 \mu \mathrm{g} \mathrm{mL}{ }^{-1}$, at $37^{\circ} \mathrm{C}$ for 24 hours. (A) Multiphoton photoluminescence imaging $(20 \times 10.75$ N.A.) of A549 cells and KC incubated with MWNT- $\mathrm{NH}_{3}{ }^{+}$and stained with Alexa Fluor $^{\circledR} 488$ phalloidin (actin filament stain) and DAPI (nuclear stain). Imaging was carried out with $950 \mathrm{~nm}$ excitation for $\mathrm{MWNT}^{-\mathrm{NH}_{3}}{ }^{+}$and with $750 \mathrm{~nm}$ excitation for both Alexa Fluor ${ }^{\mathbb{R}} 488$ phalloidin and DAPI Emission filters of 595-665 nm, 500-524 nm or 430-490 nm were used for CNTs (red), Alexa Fluor ${ }^{\circledR} 488$ phalloidin (green) or DAPI (blue), respectively. (B) Single-photon intensity and multi-photon intensity and lifetime images of $\mathrm{KC}$ incubated with $\mathrm{MWNT}-\mathrm{NH}_{3}{ }^{+}$. From left to right: singlephoton intensity (CCD), multiphoton intensity, intensity contribution from long lifetimes (fluorophores), intensity contribution from short lifetime $\left(\right.$ MWNT- $\mathrm{NH}_{3}{ }^{+}$), average fluorescence lifetime. MWNT- $\mathrm{NH}_{3}{ }^{+}$does not emit with single-photon excitation. Using multiphoton excitation, its short lifetime allows temporal unmixing from conventional fluorophores. All images field of views are $160 \mu \mathrm{m}$.

at half maximum (FWHM) value of $1.09 \mu \mathrm{m}$. Larger aggregates were observed in the peri-nuclear region. On the other hand, A549 cells showed CNT clusters with a FWHM of $0.76 \mu \mathrm{m}$
(Fig. S5B, ESI $\dagger$ ). The differences in cluster sizes could be due to differences in internalization pattern of the different cell types, i.e., ability of phagocytosis.

The main disadvantage of using CNTs to image biological samples is their relatively broad PL emission spectra, and as such, bleeding of the PL signal into the other fluorescence channels can become detrimental. However, using lifetime microscopy, the fast PL decay of CNTs can be efficiently separated from the generally long lifetime of common fluorophores. Using a conventional bi-exponential fit model, ${ }^{12}$ the contribution of short $(<0.2 \mathrm{~ns})$ and long ( $>0.5 \mathrm{~ns}$ ) lifetime components $\left(A_{1}\right.$ and $A_{2}$ respectively) can be retrieved with remarkable ease. Fig. $2 \mathrm{~B}$ shows that control KCs (no f-MWNT) display no short lifetime components in both blue and green channels, leading to an average lifetime of $1.8 \pm$ $0.1 \mathrm{~ns}$ for DAPI and $1.1 \pm 0.2 \mathrm{~ns}$ for Alexa Fluor 488 phalloidin. KC incubated with MWNT- $\mathrm{NH}_{3}{ }^{+}$shows a strong short lifetime contribution in all channels while only the blue and green channels shows a long lifetime contribution (DAPI and Alexa Fluor 488). It is worth noting that the f-MWNT do not produce a measurable PL signal using single-photon excitation (CCD images).

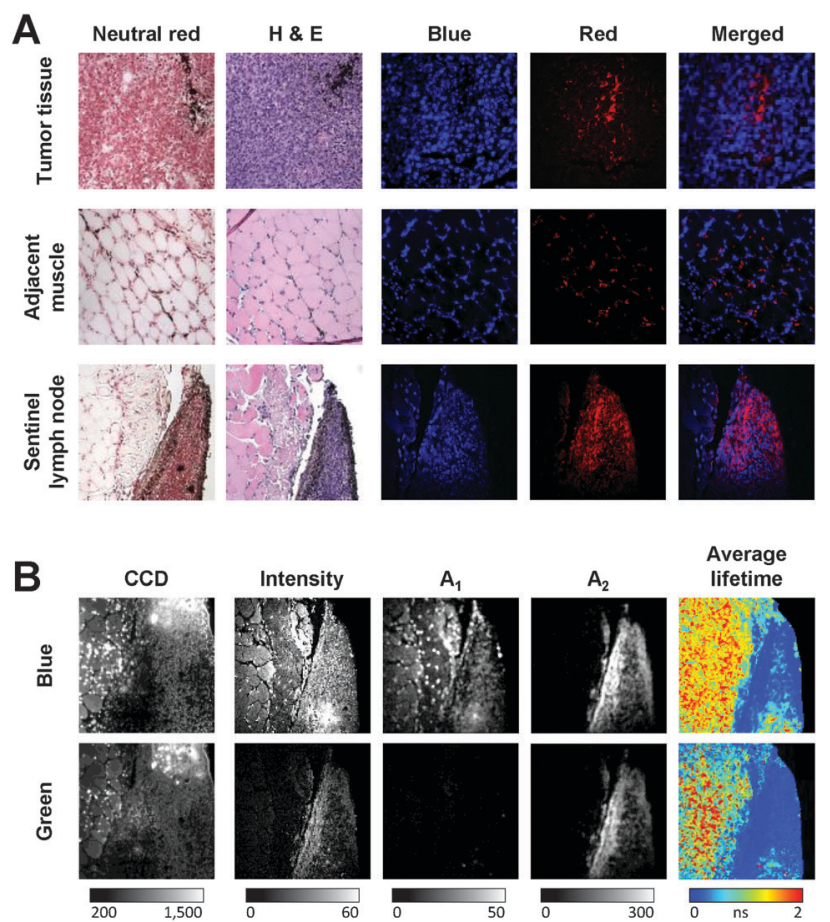

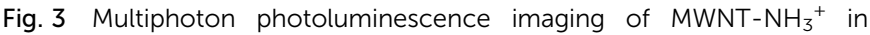
tumors. (A) Multiphoton luminescence imaging of tumors. CT-26 tumorbearing mice were injected intra-tumorally with $50 \mu \mathrm{g} / 50 \mu \mathrm{L}$ of MWNT$\mathrm{NH}_{3}{ }^{+}$. Tumors were excised, fixed, sectioned and stained with DAPI (for photoluminescence imaging), or processed for histological examination by neutral red and H\&E staining. Three major histological regions were identified: the tumor mass, adjacent muscle and a sentinel lymph node. In neutral red and $\mathrm{H} \& \mathrm{E}$ stained sections, MWNT- $\mathrm{NH}_{3}{ }^{+}$appeared as black clusters, which colocalized with the red signals in the red photoluminescent channel. (B) Lifetime imaging of the sentinel lymph node. From left to right: single-photon intensity (CCD), multiphoton intensity, intensity contribution from long lifetimes (fluorophores), intensity contribution from short lifetime (MWNT- $\mathrm{NH}_{3}{ }^{+}$), average fluorescence lifetime. All images field of views are $160 \mu \mathrm{m}$. 
Imaging f-MWNT in complex tissue environment is more challenging so f-MWNT was injected intra-tumorally (50 $\mu \mathrm{g} / 50 \mu \mathrm{l})$ in a subcutaneous CT-26 colon cancer solid tumor, implanted at the lower flanks of Balb/c mice. Tumors $\left(600 \mathrm{~mm}^{3}\right)$ were excised, fixed and processed for histology. Neutral red and H\&E staining of $10 \%$ neutral buffered formalin fixed tumors showed three distinctive regions: tumor mass, an adjacent muscle tissue and a sentinel lymph node (Fig. 3A, panels from top to bottom). Tumor sections from the same region were de-waxed and sectioned to $\sim 30 \mu \mathrm{m}$ thick slices. The nuclei were stained with DAPI, and the samples were imaged as described for in vitro samples. Images of the DAPIstained nuclei (blue channel), MWNT- $\mathrm{NH}_{3}{ }^{+}$(red channel) and the merged channels are shown in Fig. 3A (right). MWNT- $\mathrm{NH}_{3}{ }^{+}$cannot only be seen in the tumor mass but also draining into an adjacent sentinel lymph node. This is important from biomedical point of view as finding theranostic multiphoton imaging probes is of utmost importance. f-MWNT were also imaged in intact zebrafish (Fig. S6, ESI $\dagger$ ). Similarly, lifetime microscopy was performed on the sentinel lymph node sections and both short and long lifetime contributions were calculated (Fig. 3B). While similar short lifetime contributions from the f-MWNT were observed in both blue and green channels, only the fluorescence from DAPI in the blue channel was observed in the long lifetime component.

\section{References}

1 R. Pomraenke, S. Reich and J. Maultzsch, Phys. Status Solidi B, 2006, 243, 2428.

2 K. T. Al-Jamal, F. M. Toma, A. Yilmazer, H. Ali-Boucetta, A. Nunes, M. A. Herrero, B. Tian, A. Eddaoudi, W. T. Al-Jamal, A. Bianco, M. Prato and K. Kostarelos, FASEB J., 2010, 24, 4354.

3 H. Ali-Boucetta, K. T. Al-Jamal, D. McCarthy, M. Prato, A. Bianco and K. Kostarelos, Chem. Commun., 2008, 459.

4 A. Nunes, N. Amsharov, C. Guo, J. Van den Bossche, P. Santhosh, T. K. Karachalios, S. F. Nitodas, M. Burghard and K. Kostarelos, Small, 2010, 6, 2281.

5 J. E. Podesta, K. T. Al-Jamal, M. A. Herrero, B. Tian, H. Ali-Boucetta, V. Hegde, A. Bianco, M. Prato and K. Kostarelos, Small, 2009, 5, 1176.

6 M. E. Brennan, J. N. Coleman, A. Drury, B. Lahr, T. Kobayashi and W. J. Blau, Opt. Lett., 2003, 28, 266.

7 V. Georgakilas, K. Kordatos, M. Prato, D. M. Guldi, M. Holzinger and A. Hirsch, J. Am. Chem. Soc., 2002, 124, 760.

8 S. Banerjee, S. T. Hemraj-Benny and S. S. Wong, Adv. Mater., 2005, $17,17$.

9 P. Chakravarty, R. Marches, N. S. Zimmerman, A. D.-E. Swafford, P. Bajaj, I. H. Musselman, P. Pantano, R. K. Draper and E. S. Vitetta, Proc. Natl. Acad. Sci. U. S. A., 2008, 105, 8697.

10 Y. Hashida, H. Tanaka, S. Zhou, S. Kawakami, F. Yamashita, T. Murakami, T. Umeyama, H. Imahori and M. Hashida, J. Controlled Release, 2014, 173, 59.

11 K. T. Al-Jamal and K. Kostarelos, Methods Mol. Biol., 2010, 625, 123. 12 P. R. Barber, I. D. C. Tullis, G. P. Pierce, R. G. Newman, J. Prentice, M. I. Rowley, D. R. Matthews, S. M. Ameer-Beg and B. Vojnovic, J. Microsc., 2013, 251, 154. 